\title{
Aortic root dimension changes during systole and diastole: evaluation with ECG-gated multidetector row computed tomography
}

\author{
Linda M. de Heer • Ricardo P. J. Budde • \\ Willem P. Th. M. Mali • Alexander M. de Vos • \\ Lex A. van Herwerden · Jolanda Kluin
}

Received: 8 December 2010/Accepted: 16 February 2011/Published online: 27 February 2011

(C) The Author(s) 2011. This article is published with open access at Springerlink.com

\begin{abstract}
Cardiac pulsatility and aortic compliance may result in aortic area and diameter changes throughout the cardiac cycle in the entire aorta. Until this moment these dynamic changes could never be established in the aortic root (aortic annulus, sinuses of Valsalva and sinotubular junction). The aim of this study was to visualize and characterize the changes in aortic root dimensions during systole and diastole with ECG-gated multidetector row computed tomography (MDCT). MDCT scans of subjects without aortic root disease were analyzed. Retrospectively, ECG-gated reconstructions at each $10 \%$ of the cardiac cycle were made and analyzed during systole (30-40\%) and diastole (70-75\%). Axial planes were reconstructed at three different levels of the aortic root. At each level the maximal and its perpendicular
\end{abstract}

L. M. de Heer $(\varangle) \cdot$ L. A. van Herwerden · J. Kluin Department of Cardio-Thoracic Surgery, Division of Heart and Lungs, University Medical Center Utrecht, P.O. Box 85500, 3508 GA Utrecht, The Netherlands e-mail: L.M.deheer-2@umcutrecht.nl

R. P. J. Budde · W. P. Th. M. Mali

Department of Radiology, Imaging Division, University Medical Center Utrecht, Utrecht,

The Netherlands

A. M. de Vos

Department of Cardiology, Division of Heart and Lungs, University Medical Center Utrecht, Utrecht,

The Netherlands luminal dimension were measured. The mean dimensions of the total study group $(\mathrm{n}=108$, mean age $56 \pm 13$ years) do not show any significant difference between systole and diastole. The individual dimensions vary up to $5 \mathrm{~mm}$. However, the differences range between minus $5 \mathrm{~mm}$ (diastolic dimension is greater than systolic dimensions) and $5 \mathrm{~mm}$ (vice versa). This variability is independent of gender, age, height and weight. This study demonstrated a significant individual dynamic change in the dimensions of the aortic root. These results are highly unpredictable. Most of the healthy subjects have larger systolic dimensions, however, some do have larger diastolic dimensions.

Keywords Aortic valve - Electrocardiogram (ECG)-gated imaging techniques - Multidetector row computed tomography

\section{Introduction}

Cardiac pulsatility and aortic compliance may result in aortic area and diameter changes throughout the cardiac cycle in the healthy and diseased human ascending, descending and abdominal aorta, as visualized by computed tomographic angiography (CTA), magnetic resonance angiography (MRA) and M-mode ultrasound [1-4]. The presence of dimensional changes in the aortic root (composed of the aortic 
valve annulus, the site of attachment of the aortic valve leaflets; the sinuses of Valsalva and the sinotubular junction [5]) has been extensively studied over the past few years. No dynamic changes could be observed in the human aortic root in three different studies [6-8]. However, in dogs the commissural diameter of the aortic root increased by $12 \%$ between systole and diastole [9]. Furthermore, finite element model analysis [10] showed enlarged dimensions of the aortic root during the systolic phase of the cardiac cycle. These conflicting findings have resulted in an ongoing discussion about the dynamic morphological changes in the aortic root $[11,12]$. According to the observed dynamic changes at the level of the ascending, descending and abdominal aorta, we hypothesize that these changes are present at the level of the aortic root as well. However, even in the ascending, descending and abdominal aorta it is unknown in which phase of the cardiac cycle (systole or diastole) the dimensions are the largest.

The purpose of this study was to utilize ECG-gated multidetector row computed tomography (MDCT) and characterize the normal aortic root dimensions on an individual level and evaluate dynamic changes of these dimensions during systole and diastole.

\section{Methods}

Patient selection and study design

The picture and archiving system (PACS) of the department of Radiology in the University Medical Center Utrecht was searched for patients that had underwent a contrast enhanced retrospective ECGgated MDCT of the heart from January 2006 to December 2008. The majority of these patients were part of the GROUND study [13]. The study is in compliance with the Helsinki Declaration and the local ethics committee gave their approval. Participating patients have signed an informed consent form. Data on patient body weight, body length and heart rate during the scan were retrieved from the hospital information system or the scan data. Patients with valvular heart disease, coronary heart disease, cardiomyopathy or atrial fibrillation were excluded. Valvular heart disease was defined as the presence of aortic root disease or mitral valve disease (both stenosis and regurgitation). Furthermore, in all subjects we evaluated the heart valves on the CT images for morphological abnormalities to make sure that there were no valve calcifications. Trivial tricuspid regurgitation is frequently detected by echocardiography in normal subjects [14] and these subjects are therefore not excluded in this study.

\section{Imaging protocol}

Patients were scanned on a 64- or 256-slice scanner (Brilliance 64 and iCT, respectively, Philips, Philips Medical Systems, Best, The Netherlands). Non-ionic, iodinated contrast agent (Ultravist, Bayer Healthcare Tarrytown, New York) was continuously injected intravenously followed by a saline chaser. Total contrast dose and injection rate were calculated individually based on patient weight and scan duration. The contrast media dose ranged from $85 \mathrm{ml}(300 \mathrm{mg} / \mathrm{ml}$ iodine) with an injection rate of $5.3 \mathrm{ml} / \mathrm{s}$ in patients below $70 \mathrm{~kg}$ till $107 \mathrm{ml}(300 \mathrm{mg} / \mathrm{ml}$ iodine $)$ with an injection rate of $6.7 \mathrm{ml} / \mathrm{s}$ in patients above $85 \mathrm{~kg}$. CT scanning was based on a retrospectively ECG-gated data acquisition with $64 \times 0.625 \mathrm{~mm}$ or $128 \times$ $0.625 \mathrm{~mm}$ collimation, a scan pitch of $0.16-0.2$, and a gantry rotation time of $270-420 \mathrm{~ms}$. Exposure parameters were $120 \mathrm{kVp}$ tube voltage and 500-700-mAs effective tube current, depending on patient size. The radiation dose per individual measures approximately 10 millisievert. If heart rate was $>60$ beats per minute, short-acting beta-blockers (metoprolol 5-20 mg) were administered intravenously to decrease heart rate and improve image quality.

From the acquired scan data the diastolic $(70-75 \%)$ and systolic (30-40\%) phase (0.9 mm-thick sections at $0.7-\mathrm{mm}$ intervals) were reconstructed. According to the literature, $30-40$ and $70-75 \%$ of the R-R interval represents the systolic and diastolic phase, respectively [6]. The scan range was set from the proximal aortic arch to the diaphragm.

\section{Data analysis}

Images were transferred to a dedicated workstation (Brilliance Workspace, Philips, Philips Medical Systems, Best, The Netherlands) and loaded into the cardiac viewer application for analysis. The cardiac viewer software allows three perpendicular axes to be adjusted simultaneously to obtain 3 perpendicular imaging planes. 
The luminal diameters of the aortic valve (AV) annulus, sinuses of Valsalva, and the sinotubular junction (STJ) were measured in both systole and diastole by a single observer (LMH). In this study, the image reconstruction protocol suggested by Schultz et al. [15] was used. Axial cuts through the aortic root were obtained by aligning the three perpendicular imaging planes (one axial and two longitudinal, respectively, oblique sagittal and oblique coronal; Fig. 1). To identify the true short axis, one imaging plane was aligned parallel to the AV annulus. The alignment with the AV annulus was checked in the other imaging planes. The definition of the AV annulus is controversial. From anatomical and surgical perspective, the valve leaflets are attached within the cylinder of the root in the form of a coronet [5]. According to Anderson et al. [5] proper values of the annular dimension can only be provided when measurements are made at the bottom of the leaflet attachments. Therefore, the level of the aortic valve annulus on CT is defined as the lowest level of insertion of the valve leaflets into the aortic root. The oblique sagittal plane was then modified by defining it in the axial window at the level of leaflet coaptation as the line running through the commissure and along the coaptation line of the left and non-coronary cusps thereby dividing the right coronary sinus into visually equal halves. The oblique coronal plane was defined as the line orthogonal to and crossing the oblique sagittal at the point of central leaflet coaptation. Subsequently, measurements of the root were performed at three levels on the appropriate in plane slices.
The maximal diameter (Dmax) at this level was measured in the in plane images. Perpendicular to this line, a second short axis diameter was measured (Dmin). According to Tops et al. [6] the annulus was defined as oval if the difference between these two measurements was greater than $3 \mathrm{~mm}$. For the sinuses of Valsalva the diameter was measured as a line extending from each of the three commissures to the middle of the opposite coronary cusp. The Dmax and minimal diameter were used for analysis. The STJ was measured at the junction of the sinuses and the tubular portion of the ascending aorta (Fig. 2). The differences between systolic and diastolic dimensions were determined (systolic minus diastolic dimension) and plotted in a histogram to examine the distribution of the variance.

The diameter of the AV annulus, STJ and sinuses of Valsalva was also measured on the oblique coronal view (Fig. 2). The Dmax and Dmin on the double oblique transverse view and the diameter on the coronal view were compared, resembling the comparison between 3D and 2D measurements, respectively.

\section{Statistical analysis}

All values presented are the mean \pm standard deviation for continuous variables and the percentage of total patients for categorical variables. The different diameter measurements were all normally distributed and differences between maximal and perpendicular diameters and between systole and diastole were compared in the same patient using the paired sample $t$ test. The differences between systole
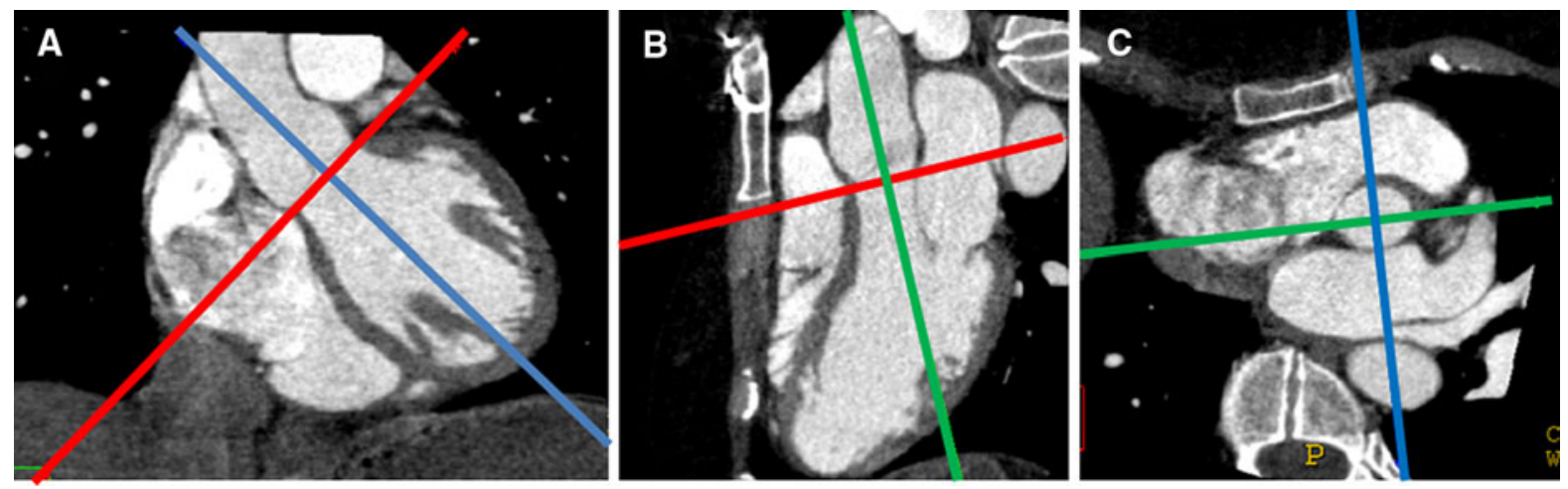

Fig. 1 Definition of the imaging planes on MDCT. The red line was aligned parallel to the valve annulus in the coronal (a) and sagittal (b) plane to obtain an in plane image of the aortic valve annulus (c). Dimension measurements (maximal and perpendicular) were made on image $\mathbf{c}$ 

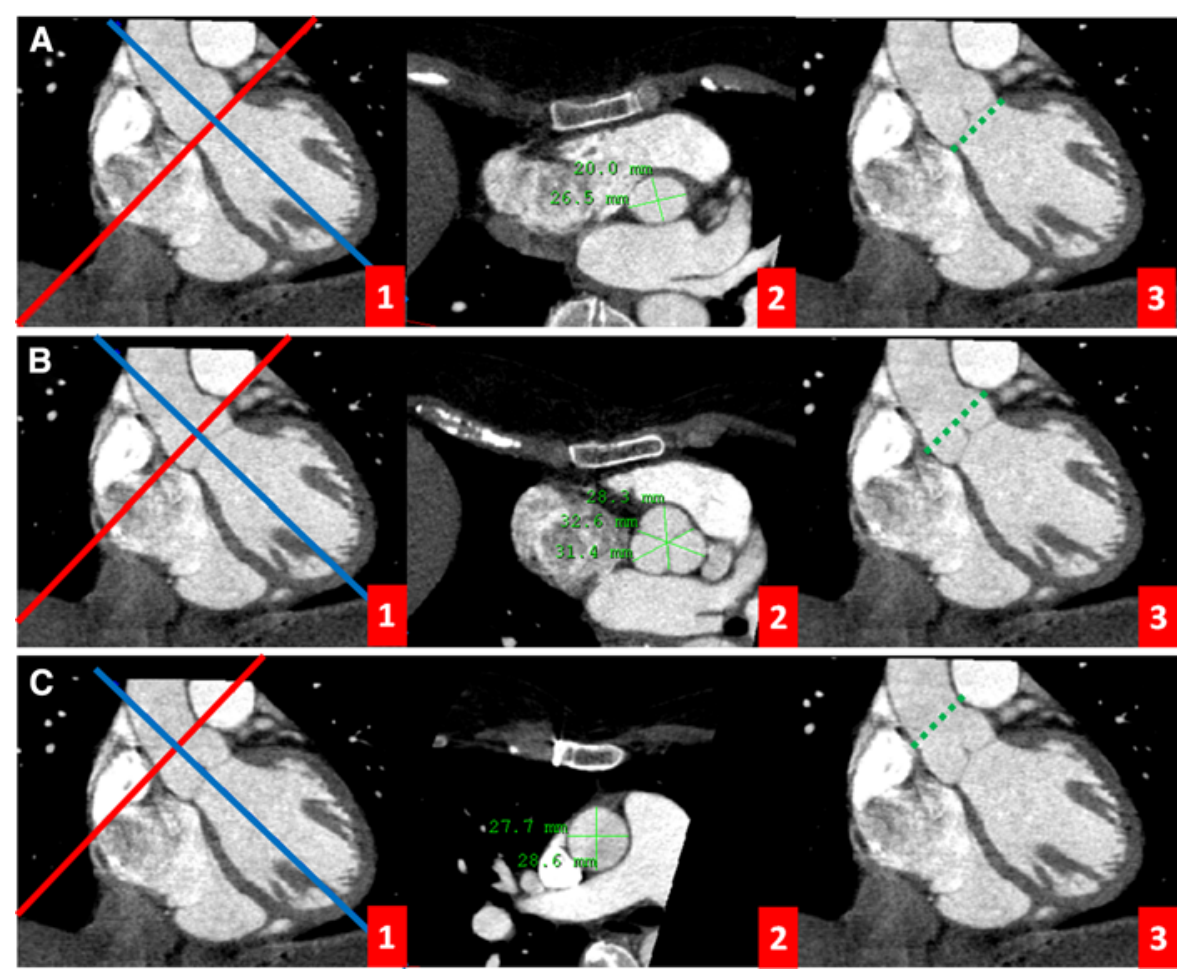

Fig. 2 The aortic annulus or base of the native leaflets was defined as the axial plane just beneath the most caudal attachment of all three aortic leaflets (a1). The largest diameter and the diameter perpendicular to this line were measured on the axial image (a2). On the oblique coronal view, the annulus measurement is shown in $\mathbf{a 3}$. The sinus of Valsalva dimensions were measured as the line extending from each commissure to the middle of the opposite coronary cusp (b2). On the oblique

and diastole were also plotted in a histogram to examine the distribution of the variance. The independent sample $t$ test was used for comparison of continuous and categoric variables, as appropriate. All $p$ values were 2-sided. Calculations were performed with the Statistical Package for the Social Sciences version 15.0 (SPSS Inc.Chicago, IL). All statistical tests were 2 -sided and a $p$ value $<0.05$ was considered statistically significant.

\section{Results}

We enrolled a total of 108 subjects (89 males, 19 females) with a mean age of $56.1 \pm 12.5$ years. The baseline characteristics, including a gender sub analysis, are demonstrated in Table 1. coronal view, the sinus of Valsalva measurement is shown in b3. The sinotubular junction was measured at the junction of the sinuses of Valsalva and the tubular portion of the ascending aorta (c1). The largest diameter and the diameter perpendicular to this line were measured on the axial image (c2). On the oblique coronal view, the sinotubular junction measurement is shown in $\mathbf{c 3}$

The overall image quality of the 108 MDCT-scans used for this study was good. Scans with severe artifacts were excluded.

\section{Aortic root dimensions}

There was a significant difference between the mean Dmax and mean Dmin at all levels (AV annulus, sinuses of Valsalva and STJ) (Table 2). The mean difference between the Dmax and Dmin of the AV annulus in systole and diastole was $4.6 \pm 2.5$ and $6.0 \pm 2.6 \mathrm{~mm}$, respectively. Sixty-seven subjects (70.3\%) had an oval shaped root (defined as a $\geq 3 \mathrm{~mm}$ difference between Dmax and Dmin) during both systole and diastole. Two subjects (1.9\%) had only an oval shaped annulus during systole and 19 subjects $(17.6 \%)$ only during diastole. Eleven subjects $(10.2 \%)$ had non-oval shaped annulus. The 
Table 1 Baseline patient characteristics

\begin{tabular}{lllll}
\hline Parameter & Study population $\mathrm{N}=108$ & Male $\mathrm{N}=89$ & Female $\mathrm{N}=19$ & $p$ value \\
\hline Age (years)* & $56.1 \pm 12.5$ & $55.6 \pm 11.6$ & $58.2 \pm 16.2$ & NS \\
Male gender (\%) & $89(82)$ & - & - & - \\
Body length $(\mathrm{cm}) *$ & $176 \pm 8$ & $179 \pm 6$ & $168 \pm 6$ & $<0.01$ \\
Body weight $(\mathrm{kg}) *$ & $82 \pm 13$ & $85 \pm 12$ & $72 \pm 14$ & 0.01 \\
Heart rate during imaging (beats/min)* & $59 \pm 10$ & $55 \pm 9$ & $69 \pm 6$ & $<0.01$ \\
\hline
\end{tabular}

* Values are displayed as mean $\pm \mathrm{SD}$

Table 2 A comparison of the mean maximal (Dmax) and perpendicular (Dmin) diameters at three levels of the aortic root

\begin{tabular}{|c|c|c|c|c|c|c|}
\hline \multirow[t]{2}{*}{ Level of measurement } & \multicolumn{2}{|l|}{ Systolic } & \multirow[t]{2}{*}{$p$ value } & \multicolumn{2}{|l|}{ Diastolic } & \multirow[t]{2}{*}{$p$ value } \\
\hline & Dmax & Dmin & & Dmax & Dmin & \\
\hline Aortic valve annulus & $29.7 \pm 3.4$ & $25.1 \pm 3.3$ & 0.000 & $30.1 \pm 3.0$ & $24.0 \pm 3.1$ & 0.000 \\
\hline Sinuses of Valsalva* & $36.4 \pm 4.0$ & $33.5 \pm 3.6$ & 0.000 & $35.4 \pm 3.8$ & $32.8 \pm 3.6$ & 0.000 \\
\hline Sinotubular junction & $32.4 \pm 3.8$ & $30.0 \pm 3.6$ & 0.000 & $31.5 \pm 3.9$ & $29.5 \pm 3.5$ & 0.000 \\
\hline
\end{tabular}

* The diameter of the sinuses of Valsalva is measured in three directions. The maximal diameter and the minimal diameter were shown as Dmax and Dmin, respectively

mean Dmax and Dmin of all 108 subjects together showed no significant differences between systole and diastole (Table 2). The Dmax of the AV annulus in systole versus diastole was $29.7 \pm 3.4$ versus $30.1 \pm 3.0 \mathrm{~mm}$ and the Dmin $25.1 \pm 3.3$ versus $24.0 \pm 3.1 \mathrm{~mm}$, respectively. The Dmax of the sinuses of Valsalva was $36.4 \pm 4.0 \mathrm{~mm}$ during systole and $35.4 \pm 3.8 \mathrm{~mm}$ during diastole and the Dmin $33.5 \pm 3.6$ versus $32.8 \pm 3.6 \mathrm{~mm}$, respectively. At the level of the STJ the Dmax was $32.4 \pm 3.8$ versus $31.5 \pm 3.9 \mathrm{~mm}$ during systole and diastole, respectively and the Dmin was $30.0 \pm 3.6$ and $29.5 \pm 3.5 \mathrm{~mm}$, respectively.

The SPSS paired sample $t$ test results showed a significant difference between the systolic and diastolic diameters in all individual subjects $(<0.001)$ which is absent when the mean diameters were summed up, because the distribution of these differences is variable at all levels, as is shown in the histograms in Fig. 3. In most of the subjects the systolic diameters of the AV annulus, the sinuses of Valsalva and the STJ were larger than the diastolic dimensions (Table 3).

\section{Gender differences}

The diameters, both Dmax and Dmin, of the male aortic root were significantly larger than the diameters of the female aortic root (Table 4). There is no significant correlation between length of the subjects and the dimensions and weight of the subjects and the dimensions (data not shown).

\section{$3 \mathrm{D}$ versus $2 \mathrm{D}$ measurements}

Table 5 shows a significant difference between both the in plane Dmax and Dmin and the longitudinal "one direction" coronal measurement, indicating an oval shaped aortic root.

\section{Discussion}

In the present study, ECG-gated MDCT (64- and 256 slice) was used to measure the dimensions of the aortic root (AV annulus, sinuses of Valsalva and STJ) in 108 healthy subjects. There was a significant difference between these three-dimensional measurements and the two-dimensional measurements, represented by the coronal measurement on MDCT. Other studies confirmed these results [6, 7].

With MDCT, previous studies did not show any significant difference between the systolic and diastolic dimensions of the aortic root. This unexpected finding might be caused by the method used to measure the dimensions. In these studies the 


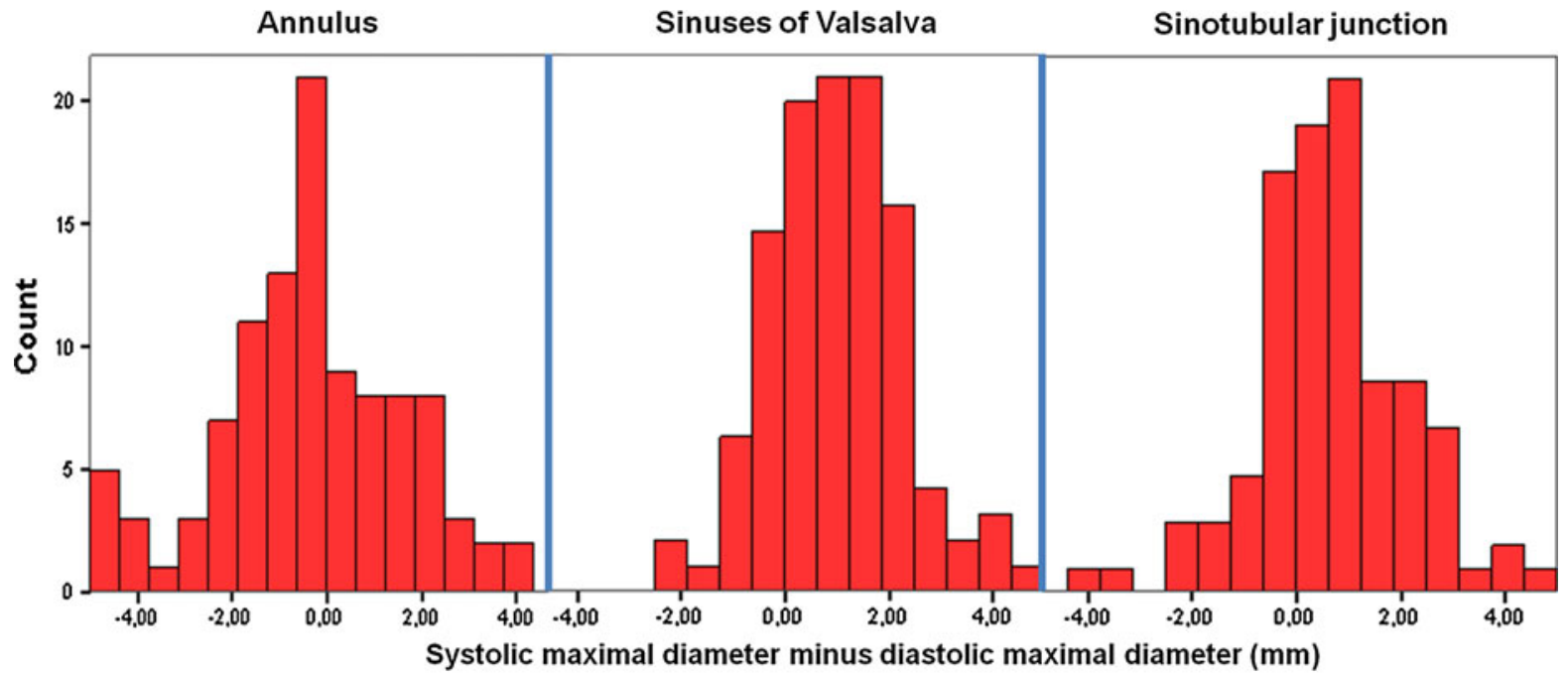

Fig. 3 Histograms of the difference between the maximal systolic diameter and the maximal diastolic diameter at the level of the aortic valve annulus (a), sinuses of Valsalva (b) and sinotubular junction (c), showing a highly variable distribution

Table 3 The absolute differences and the distribution of the differences between the systolic and diastolic dimensions in the individual patients at all levels

\begin{tabular}{|c|c|c|c|c|c|c|c|c|}
\hline \multirow[t]{2}{*}{ Level } & \multirow[t]{2}{*}{ Dmax* } & \multirow[t]{2}{*}{ Dmin* } & \multicolumn{2}{|c|}{ Systolic $>$ diastolic } & \multicolumn{2}{|c|}{ Systolic $=$ diastolic } & \multicolumn{2}{|c|}{ Diastolic $>$ systolic } \\
\hline & & & Dmax & Dmin & Dmax & Dmin & Dmax & Dmin \\
\hline Annulus & $-0.3 \pm 2.4$ & $1.1 \pm 2.0$ & 38.5 & 69.2 & 2.8 & 0 & 58.7 & 30.8 \\
\hline Sinuses* & $0.8 \pm 2.0$ & $0.6 \pm 1.4$ & 78.5 & 76.6 & 1.9 & 5.6 & 19.6 & 17.8 \\
\hline Sinotubular & $1.0 \pm 1.4$ & $0.8 \pm 1.6$ & 65.0 & 61.1 & 3.9 & 1.9 & 31.1 & 37.0 \\
\hline
\end{tabular}

Values are displayed as percentage of the total number of subjects

Dmax $=$ maximal diameter

Dmin $=$ perpendicular diameter

* Values are displayed as mean \pm standard deviation

dimensions during systole and diastole were measured in coronal and single oblique sagittal $[6,7]$ and in longitudinal reconstructions [8]. In our study, the axial reconstruction was used to measure the dimensions at different levels in the aortic root.

A comparison between the Dmax and Dmin on the axial image revealed a significant difference between both values that was variable between subjects. Seventy-six subjects $(70.3 \%)$ had an oval shaped root (defined as a $\geq 3 \mathrm{~mm}$ difference between Dmax and Dmin [6]) during both systole and diastole. Two subjects (1.9\%) had just an oval shaped annulus during systole and 19 subjects (17.6\%) just during diastole. 11 subjects $(10.2 \%)$ had a more circular shaped annulus. The fact that the aortic valve annulus is an oval shaped structure, rather than a circle, is confirmed by other studies $[6,7]$. The difference in shape between systole and diastole implies a change in dimensions during the cardiac cycle.

Although many studies showed dynamic changes in the aorta diameter at different levels during the cardiac cycle, the presence of systolic and diastolic changes at the level of the aortic root were not observed in previous human studies by noninvasive imaging with MDCT [6-8]. Sonomicrometry studies in dogs [9] and finite element model analysis [10] did show enlargement of the aortic root dimensions during systole compared to diastole. There is an ongoing discussion [12] regarding the mechanism by which the aortic root dilates prior to opening of the 
Table 4 Aortic root dimensions: stratification by gender and comparison between male and female

\begin{tabular}{llll}
\hline Variable & Male N $=89$ & Female N $=19$ & $p$ value \\
\hline Aortic valve annulus & & & \\
Systolic Dmax & $30.4 \pm 3.0$ & $26.3 \pm 3.5$ & 0.000 \\
Diastolic Dmax & $30.7 \pm 2.7$ & $27.2 \pm 2.8$ & 0.000 \\
Systolic Dmin & $25.6 \pm 3.0$ & $22.4 \pm 3.3$ & 0.001 \\
Diastolic Dmin & $24.6 \pm 2.8$ & $21.0 \pm 2.6$ & 0.000 \\
Sinuses of valsalva & & & \\
Systolic Dmax & $37.1 \pm 3.8$ & $32.7 \pm 2.7$ & 0.000 \\
Diastolic Dmax & $36.0 \pm 3.6$ & $32.4 \pm 2.8$ & 0.000 \\
Systolic Dmin & $34.2 \pm 3.4$ & $29.9 \pm 2.6$ & 0.000 \\
Diastolic Dmin & $33.4 \pm 3.4$ & $29.9 \pm 3.1$ & 0.000 \\
Sinotubular junction & & & \\
Systolic Dmax & $32.9 \pm 3.8$ & $29.8 \pm 2.9$ & 0.001 \\
Diastolic Dmax & $31.9 \pm 3.8$ & $29.4 \pm 3.7$ & 0.018 \\
Systolic Dmin & $30.5 \pm 3.5$ & $27.7 \pm 3.1$ & 0.003 \\
Diastolic Dmin & $29.9 \pm 3.5$ & $27.4 \pm 3.6$ & 0.002 \\
\hline
\end{tabular}

All values are displayed as mean $\pm \mathrm{SD}$

Dmax $=$ maximal diameter

Dmin $=$ perpendicular diameter

aortic valve. Can this dilatation be explained by passive fluid dynamics or is it an active process and are there physiological or pathological processes that can influence this dilatation?

Angiography and echocardiography are helpful imaging modalities in the visualization of the aortic root. With the advent of transcatheter AV implantation procedures (TAVI) [16-18], the closed chest imaging becomes even more important, because of the difficulty with prosthesis sizing, accurate positioning of the prosthesis, the covering of the coronary ostia by the prosthesis [18] and even occlusion of the left coronary artery [17]. Currently used angiography and echocardiography are limited by their twodimensional character [6-8]. MDCT can provide three-dimensional images with a high spatial resolution and give detailed information on the anatomy of the aortic root and the relation of the annulus with the coronary arteries [6]. The use of real-time 3D TEE to guide cardiac interventions has increased over the last few years. Direct planimetry of the aortic annular area by 3D TEE volumetric imaging showed the best agreement with MDCT as a 'gold standard' [19], although it still underestimated the MDCT planimetered areas by up to $9.6 \%$, most likely due to a lower spatial resolution associated with 3D TEE volumetric imaging [19].

To demonstrate differences between systolic and diastolic dimensions, an individual comparison between both values was made. This comparison revealed significant dynamic changes in the aortic root dimensions. When the individual measurements were added to calculate the mean of the entire study population, this dynamic difference disappeared, as was the case in the other studies [6-8]. This can be explained by the highly variable distribution of the difference between systolic and diastolic dimensions, as was shown in the histogram. As expected, most of the individual subjects had larger systolic dimensions, compared to the diastolic dimensions, however, some

Table 5 Comparison between 3D (axial) and 2D (coronal) dimension measurements

\begin{tabular}{|c|c|c|c|c|c|}
\hline \multirow[t]{2}{*}{ Mean diameter (mm) } & \multirow[t]{2}{*}{ Coronal } & \multicolumn{4}{|l|}{ Axial } \\
\hline & & Dmax & $p$ value & Dmin & $p$ value \\
\hline \multicolumn{6}{|l|}{ Systolic } \\
\hline Aortic valve annulus & $26.6 \pm 2.8$ & $29.7 \pm 3.4$ & 0.000 & $25.1 \pm 3.3$ & 0.000 \\
\hline Sinuses of Valsalva & $37.1 \pm 3.8$ & $36.4 \pm 4.0$ & 0.000 & $33.5 \pm 3.6$ & 0.000 \\
\hline Sinotubular junction & $29.4 \pm 3.7$ & $32.4 \pm 3.8$ & 0.000 & $30.0 \pm 3.6$ & 0.002 \\
\hline \multicolumn{6}{|l|}{ Diastolic } \\
\hline Aortic valve annulus & $26.9 \pm 2.4$ & $30.1 \pm 3.0$ & 0.000 & $24.0 \pm 3.1$ & 0.000 \\
\hline Sinuses of Valsalva & $36.5 \pm 3.9$ & $35.4 \pm 3.8$ & 0.000 & $32.8 \pm 3.6$ & 0.000 \\
\hline Sinotubular junction & $28.9 \pm 3.7$ & $31.5 \pm 3.9$ & 0.000 & $29.5 \pm 3.5$ & 0.001 \\
\hline
\end{tabular}

All values are displayed as mean $\pm \mathrm{SD}$

Dmax $=$ maximal diameter

Dmin $=$ perpendicular diameter 
had larger diastolic dimensions. According to these results we can conclude that there are dynamic changes in the aortic root of healthy subjects, however, they are very unpredictable.

With the limited available literature on this subject, an explanation of this unpredictability is challenging. Most of the studies describing the aortic root dimensions were carried out either by models or at autopsy [20,21]. Observations of the aortic root in the beating heart has been done both indirectly and directly. Indirect methods include X-ray techniques, with either contrast material (human study) [22], radiopaque markers (animal studies in dogs and sheep) [9, 23-25] or sonomicrometry (animal studies in sheep) $[11,26]$ as well as echocardiography (animal study in dogs and a human study) [27, 28]. Direct observation of the aortic root in dogs can be achieved with cinematographic techniques (animal study in dogs), in which blood can be a disturbing factor [29] or electromagnetic induction (animal study with dogs) [30]. The studies performed in dogs and sheep with various imaging techniques all showed expansion of the aortic root during systole. A $16 \%$ radial displacement of the commissures was observed in an isolated aortic root model [21] and $12 \%$ in dogs [9]. The aortic root expansion starts prior to ejection and follows a precise order. Each root level reached maximal expansion during the first third of ejection [26]. At end-diastole, the aortic root had a truncated cone shape and during systole it became more cylindrical in a sonomicrometry study in sheep [26]. This result corresponds to our findings. However, Dagum et al. [25] described an annular contraction during the ejection phase in sheep. In summary, there is an ongoing discussion with regard to the dynamic behaviour of the aortic root. The main limitation of the animal studies is the invasive, acute and open-chest nature of the models. Furthermore, the findings in dogs and sheep are not necessarily applicable to humans. No previous studies in humans proved pure systolic expansibility of the aortic root $[6-8,28]$. The lack of dynamic behaviour of the aortic root was attributed by the authors to the lack of elasticity in the aortic root in comparison to the much younger dogs and sheep used in the animal studies [8, 28]. Howard et al. [31] has simulated stiffening of the aortic root and showed a disappearance of the radial expansion of the aortic root when stiffened.
The present study describesa non-invasive closedchest method to measure the dynamic aortic root behaviour in the axial plane with MDCT. This study showed that there is a high variability between the different individuals, without any correlation with age, body length or body weight. The mechanism of the aortic root dynamics can only be partially understood with the available data and the knowledge from literature. Better understanding of the aortic root dynamic anatomy is a necessity. Because of the high individual variability, shown in this study, an individual non-invasive determination of the dynamic characteristics of the aortic root in each subject is necessary for adequate analysis. The method used by Van Prehn et al. [32] could be an interesting method for segmentation of the different levels of the aortic root in the axial plane. With this method dynamic changes over the complete cardiac cycle in all directions can be analyzed in the thoracic aorta. In the future, this might support the cardiothoracic surgeons, cardiologists and engineers in their prosthesis selection, sizing and design.

In conclusion, the AV annulus is an oval, rather than a circular structure. Previous studies could not demonstrate dynamic changes in the aortic root, although they were present in the ascending, descending and abdominal aorta. This may be explained by the presence of a substantial variability in the dynamic changes of the aortic root dimensions during systole and diastole in healthy subjects. This study demonstrated a significant dynamic change in all subjects with a variable distribution between systole and diastole, due to the complex geometry of the aortic root. More research is needed to determine the exact shape changes in all directions throughout the entire cardiac cycle in both health and disease.

\section{Limitations}

This study showed significant differences of the aortic root dimensions, between 30 and $40 \%$ of the RR-interval on the ECG (systole) and 70-75\% of the RR-interval (diastole), however, some limitations have to be addressed.

First, the change of the aortic root diameter and radius and the area on the in plane images was only measured in two phases of the cardiac cycle. Dynamic diameter changes in multiple directions 
should be determined during the whole cardiac cycle, in each $10 \%$ of the RR-interval, to adjust the prosthesis design and choice of prosthesis diameter to assess. Furthermore the manual measurement of the aortic root diameters in this study may be less reliable than automatic computer segmentations by dedicated software. However, manual measurement is a clinically routinely used method.

The blood pressure and the cardiac output of the patient at the time of the MDCT were not measured. Both may influence the dynamic behaviour of the aortic root and may be a confounder in the presented results. Studies have showed that the aortic stiffness is increased in patients with hypertension [33, 34]. Therefore, It could be that the dynamic changes in subjects with lower blood pressures are even more pronounced.

Acknowledgments The authors gratefully acknowledge the support of The Netherlands Heart Foundation and the Smart Mix Program of the Netherlands Ministry of Economic Affairs and the Netherlands Ministry of Education, Culture and Science. Furthermore, the authors wish to thank Frederiek de Heer for her statistical assistance.

\section{Conflict of interest None.}

Open Access This article is distributed under the terms of the Creative Commons Attribution Noncommercial License which permits any noncommercial use, distribution, and reproduction in any medium, provided the original author(s) and source are credited.

\section{References}

1. Van Herwaarden JA, Bartels LW, Muhs BE et al (2006) Dynamic magnetic resonance angiography of the aneurysm neck: conformational changes during the cardiac cycle with possible consequences for endograft sizing and future design. J Vasc Surg 44:22-28

2. Van Prehn J, Vincken KL, Muhs BE et al (2007) Toward endografting of the ascending aorta: insight into dynamics using dynamic cine-CTA. J Endovasc Ther 14:551-560

3. Teutelink A, Muhs BE, Vincken KL et al (2007) Use of dynamic computed tomography to evaluate pre- and postoperative aortic changes in AAA patients undergoing endovascular aneurysm repair. J Endovasc Ther 14:44-49

4. Goergen CJ, Johnson BL, Greve JM, Taylor CA, Zarins CK (2007) Increased anterior abdominal aortic wall motion: possible role in aneurysm pathogenesis and design of endovascular devices. J Endovasc Ther 14:574-584

5. Anderson RH (2000) Clinical anatomy of the aortic root. Heart 84:670-673
6. Tops LF, Wood DA, Delgado V et al (2008) Noninvasive evaluation of the aortic root with multislice computed tomography. Implication for transcatheter aortic valve replacement. J Am Coll Cardiol Imaging 1:321-330

7. Wood DA, Tops LF, Mayo JR (2009) Role of multislice computed tomography in transcatheter aortic valve replacement. Am J Cardiol 103:1295-1301

8. Kazui T, Izumoto H, Yoshioka K, Kawazoe K (2006) Dynamic morphologic changes in the normal aortic annulus during systole and diastole. J Heart Valve Dis 15:617-621

9. Thubrikar MK, Nolan SP (1977) Normal aortic valve function in dogs. Am J Cardiol 50:563-568

10. Gnyaneshwar R, Kumar RK, Balakrishnan KR (2002) Dynamic analysis of the aortic valve using finite element model. Ann Thorac Surg 73:1122-1129

11. Pang DC, Choo SJ, Luo HH et al (2000) Significant increase of aortic root volume and commissural are occurs prior to aortic valve opening. J Heart Valve Dis 9:9-15

12. Vesely I (2000) Aortic root dilation prior to valve opening explained by passive hemodynamics. J Heart Valve Dis 9:16-20

13. De Vos AM, Rutten A, Van de Zaag-Loonen HJ et al (2008) Non-invasive cardiac assessment of high-risk patients (the GROUND study): rationale, objectives, design of a multicenter randomized controlled trial. Trials 9:49

14. Vahanian A, Baumgartner H, Bax J (2007) Guidelines on the management of valvular heart disease. Eur Heart $\mathbf{J}$ 28:230-268

15. Schultz CJ, Moelker A, Piazza N et al (2010) Three dimensional evaluation of the aortic annulus using multislice computed tomography: are manufacturer's guidelines for sizing for percutaneous aortic valve replacement helpful? Eur Heart J 31:849-856

16. Cribier A, Etchaninoff H, Tron C (2006) Treatment of calcific aortic stenosis with the percutaneous heart valve: mid-term follow-up from the initial feasibility studies: the French experience. J Am Coll Cardiol 47:1214-1223

17. Webb JG, Chandavimol M, Thompson CR (2006) Percutaneous aortic valve implantation retrograde from the femoral artery. Circulation 113:842-850

18. Grube E, Laborde JC, Gerckens U (2006) Percutaneous implantation of the CoreValve self-expanding valve prosthesis in high-risk patients with aortic valve disease: the Siegburg firstin-man study. Circulation 114:1616-1624

19. Ng ACT, Delgado V, Van der Kley F et al (2010) Comparison of aortic root dimensions and geometries before and after transcatheter aortic valve implantation by 2 - and 3-dimensional transesophageal echocardiography and multislice computed tomography. Circ Cardiovasc Imaging 3:94-102

20. Bellhouse B, Bellhouse F (1969) Fluid mechanics of model normal and stenosed aortic valves. Circ Res 25:694-704

21. Brewer RJ, Deck JD, Capati B, Nolan SP (1976) The dynamic aortic root: its role in aortic valve function. J Thorac Cardiovasc Surg 72:413-417

22. Stein PD (1971) Roentgenographic method for measurement of the cross-sectional area of the aortic valve. Am Heart J 81:622-634

23. Bernuth GV, Tsakiris AG, Wood EH (1971) Effects of variations in the strength of left ventricular contraction on aortic valve closure in the dog. Circ Res 28:705-716 
24. Thubrikar M, Bosher LP, Nolan SP (1979) The mechanism of opening of the aortic valve. J Thorac Cardiovasc Surg 77:863-870

25. Dagum P, Green GR, Nistal FJ et al (1999) Deformational dynamics of the aortic root: modes and physiological determinants. Circulation 100:II54-II62

26. Lansac E, Lim HS, Shomura Y et al (2002) A fourdimensional study of the aortic root dynamics. Eur J Cardio-thorac Surg 22:497-503

27. Laniado S, Yellin E, Terdiman R, Meytes I, Stadler J (1978) Hemodynamic correlates of the normal aortic valve echogram: a study of sound, flow and motion. Circulation 54:729-737

28. Acar C, Varnous S. In response to Kazui T, Izumoto H, Yoshioka K, Kawazoe K (2006) Dynamic morphological changes in the normal aortic annulus during systole and diastole. J Heart Valve Dis, 15:617-621

29. Van Steenhoven AA, Verlaan CWJ, Veenstra PC, Reneman RS (1986) In vivo cinematographic analysis of behavior of the aortic valve. Am J Physiol 251:H236$\mathrm{H} 241$
30. Higashidate M, Tamiya K, Beppu T, Imai Y (1995) Regulation of the aortic valve opening. In vivo dynamic measurement of the aortic valve orifice area. $\mathrm{J}$ Thorac Cardiovasc Surg 110:496-503

31. Howard IC, Patterson EA, Yoxall A (2003) On the opening mechanism of the aortic valve: some observations of simulations. J Med Eng Technol 27:259-266

32. Van Prehn J, Vincken KL, Sprinkhuizen SM et al (2009) Aortic pulsatile distention in young healthy volunteers is asymmetric: analysis with ECG-gated MRI. Eur J Vasc Endovasc Surg 37:168-174

33. Vitarelli A, Giordano M, Germano G, Pergolini M, Cicconetti P, Tomei F, Sancini A, Battaglia D, Dettori O, Capotosto L, De Cicco V (2010) De MaioM, Vitarelli M, Bruno P. Assessment of the ascending aorta wall stiffness in hypertensive patients by tissue Doppler imaging and strain Doppler echocardiography. Heart 96:1469-1474

34. Huang Y, Hu B, Huang PT, Sun HY, Zhu JA (2008) Abdominal aortic wall motion of healthy and hypertensive subjects: evaluation of tissue Doppler velocity imaging. J Clin Ultrasound 36:218-225 